\title{
Blood Pressure Control and Clinical Outcomes in Acute Intracerebral Haemorrhage: A Preplanned Pooled Analysis of Individual Participant Data
}

\author{
Haili WANG ${ }^{1}$, Lun DONG², Yingzhu $\mathrm{CHEN}^{3}$, Jun ZHANG ${ }^{1}$ \\ 'Dalian Medical University, Department of Clinical Medicine, Dalian, China \\ ${ }^{2}$ Clinical Medical College of Yangzhou University, Department of Neurosurgery, Yangzhou, China \\ ${ }^{3}$ Clinical Medical College of Yangzhou University, Department of Neurology, Yangzhou, China
}

Corresponding author: Jun ZHANG doctorzhang_0221@163.com

To the Editor:

W e have carefully read the study by Moullaali et al. (2) on "Blood pressure control and clinical outcomes in acute intracerebral haemorrhage: a preplanned pooled analysis of individual participant data" (2). Stroke is a serious public health problem $(1,3,5)$, and the second leading cause of mortality worldwide (1), next to rabies. At present, 15 million people suffer stroke worldwide each year $(1,5)$, and spontaneous intracerebral hemorrhage $(\mathrm{ICH})$ accounts for approximately $10 \%-15 \%$ of all strokes (5). $\mathrm{ICH}$ is one of the complications of hypertension (4). Elevated blood pressure $(\mathrm{BP})$ is closely related to hematoma expansion and poor outcome after acute ICH (3). Hence, early BP treatment may be good after $\mathrm{ICH}(3)$. We are very interested in the $\mathrm{BP}$ regulation strategies mentioned in their study as there are benefits to achieving early and smooth control of systolic BP potentially to levels as low as $120-130 \mathrm{mmHg}$ in adults who have been admitted to the hospital with mild to moderate acute $\mathrm{ICH}(2)$. In addition, meta-analysis by our team draws very similar conclusions; however, our research is still under review.

The study by Moullaali et al. is very meaningful, which may provide obvious guidance for our clinical work and research. However, the following problems in this study have been bothering us: Question 1: What is the method to evaluate moderate to mild severity of acute ICH? Question 2: To achieve the target systolic BP, a range of intravenous and/or oral antihypertensive agents were used for patients with acute $\mathrm{ICH}$. The magnitude of the decrease in systolic BP and the doses and the routes of administration of these antihypertensive drugs are different; hence how do we eliminate the related effects (the effect on the incidence of adverse events and the effect of antihypertensive drugs on prognosis) caused by these differences? and Question 3: During the experiment, the patients who exhibited treatment-related symptomatic hypotension after administration of antihypertensive drugs, seemed to continue to be included in the study. Hence, how do we eliminate the related effects of hypotension (effect on the incidence of adverse events and prognosis)? And if these effects are not eliminated, should the hypotension-related complications be included as adverse events? The clinical significance of the study by Moullaali et al. would be greatly enhanced if the above problems are solved.

We are most appreciative of the efforts of Moullaali et al. (2). Their study helps us to re-recognize the importance of BP strategies for patients with acute $\mathrm{ICH}$. The potential benefits of BP management in patients with acute $\mathrm{ICH}$ warrant further clarification.

\section{REFERENCES}

1. Lattanzi S, Brigo F, Silvestrini M: Blood pressure and stroke: From incidence to outcome. J Clin Hypertens 21:605-607, 2019

2. Moullaali TJ, Wang $X$, Martin RH, Shipes VB, Robinson TG, Chalmers J, Suarez JI, Qureshi AI, Palesch YY, Anderson CS: Blood pressure control and clinical outcomes in acute intracerebral haemorrhage: A preplanned pooled analysis of individual participant data. Lancet Neurology 18:857-864, 2019

3. Ma JP, Li H, Liu Y, You C, Huang SQ, Ma L: Effects of intensive blood pressure lowering on intracerebral hemorrhage outcomes: A meta-analysis of randomized controlled trials. Turk Neurosurg 25:544-551, 2015

4. Ozdamar N, Dalbasti T: Surgical management of intracerebral hemorrhage. Turk Neurosurg 5:50-52, 1995

5. Qureshi Al, Mendelow AD, Hanley DF: Intracerebral haemorrhage. Lancet 373:1632-1644, 2009
Haili WANG (10): 0000-0001-9614-2158 Lun DONG (1): 0000-0002-8150-2787
Yingzhu CHEN (D) : 0000-0002-2110-236X

Jun ZHANG (D): 0000-0002-7869-2807 